\title{
DESIGN AND MOLECULAR DOCKING STUDIES OF NOVEL ANTIMICROBIAL PEPTIDES USING AUTODOCK MOLECULAR DOCKING SOFTWARE
}

\author{
KIRANPREET KAUR, PARANJEET KAUR, AMIT MITTAL, SURENDRA KUMAR NAYAK, GOPAL L KHATIK* \\ Department of Pharmaceutical Chemistry, School of Pharmaceutical Sciences, Jalandhar, Phagwara - 144 411, Punjab, India. \\ Email: gopal.16803@lpu.co.in
}

Received: 14 July 2017, Revised and Accepted: 25 July 2017

\section{ABSTRACT}

Objective: Design of novel antimicrobial peptides (AMPs) and study through the molecular docking.

Methods: The molecular structures were drawn in ChemBiodraw ultra and by the help of ChemBiodraw 3D, all structures were energy minimized by the MM2 method and converted to pdb extension file which is readable at the AutoDock Vina (ADT) interface. The ADT 1.5.6 software is used for molecular docking purposes.

Results: Eight AMPs were designed based on the MP196 AMP. Among these KP_03R (FWRWRW-NH 2 ) showed good binding affinity. These peptides also showed the stereochemical influence on affinity toward the 3vma protein of Escherichia coli, where AMP with R stereochemistry showed better activity than its opposite stereochemistry.

Conclusion: Novel AMPs were designed by modifications on the MP196 a short chain of amino acids AMPs. Molecular docking software was used to determine the binding affinity between drug and receptor protein. Among all the designed peptides KP_03R (FWRWRW-NH 2 ) showed the maximum binding affinity against the penicillin-binding protein of $E$. coli and also exhibited stereoselective activity.

Keywords: Antimicrobial peptides, MP196, AutoDock Vina, Molecular docking.

(C) 2017 The Authors.Published by Innovare Academic Sciences Pvt Ltd. This is an open access article under the CCBY license (http://creativecommons org/licenses/by/4. 0/) DOI: http://dx.doi.org/10.22159/ajpcr.2017.v10s4.21332

\section{INTRODUCTION}

Microbial infection and its treatment is a major concern today. There increasing drug resistance to the existing antibacterial agents paved to develop the novel treatment strategies. Currently, several new approaches are adopted to manage the toxicity; multidrug resistance arises due to bacterial infections such as forgotten antibiotic, antimicrobial peptides (AMPs), vaccination, phage therapy, bacteriocins, killing factors, non-antibiotic antibacterial drugs, and quorum sensing inhibitors [1,2]. Recent studies indicate that AMPs could be a better alternative to combat the multidrug resistance infections [2]. AMPs are oligopeptides containing a varying number of amino acids from 5 to more than 100 amino acids. AMPs have a broad spectrum of activity. They target virus, fungi, bacteria, and parasite.

The rapid increase in antibiotic resistance and their short spectrum of activity lead to the emergence of AMPs. These AMPs have proved to be effective against the "superbugs". The AMPs have a broad spectrum of activity, rapid action, and show synergistic activity with other old antibiotics, AMPs, and lysozymes. AMPs block the release of cytokines by bacterial products in tissue culture and human blood and the onset of sepsis is also blocked by them in the mouse model endotoxemia [3].

The AMPs can be derived from both plants as well as animal sources. Dubos in 1939, extracted an antimicrobial agent from a soil Bacillus strain. This extract was witnessed to protect mice from Pneumococci infection [4]. In the impending years, Hotchkiss along with dubos fractionated that extract and found an AMP which was named as gramicidin [5]. Besides toxicity on the intra peritoneal application, gramicidin was proved to be effective for topical treatment of wounds and ulcers [5,6]. Moreover, in 1941 tyrocidine was discovered which was found to be effective against Gram-negative as well as Grampositive bacteria [7]. However, tyrocidine was found to cause hemolytic activity [8]. In the same year, purothionin was extracted from plant
Triticum aestivum and found to be effective against fungi and some pathogenic bacteria [9].

Apart from these plant-derived AMPs, animal-based AMPs were also discovered. The first animal derived AMP was defensin, which was extracted from rabbit leukocytes in 1956 [10]. In the impending years, bombinin from epithelia8 and lactoferrin from cow milk [11] were described. In the same year, it was proved that human leukocytes contain AMPs in their lysosomes [12]

MP196 is a hexapeptide (RWRWRW- $\mathrm{NH}_{2}$ ) which represent the small peptide with hydrophobic and positively charged amino acids as key pharmacophore depicted in (Fig. 1) $[13,14]$. MP196 is showed good activity against all type of bacteria but more effective against Grampositive bacteria including methicillin-resistant Staphylococcus aureus strains, also it found less toxic with low hemolytic activity [15]. Hence, MP196 could serve as the potential lead for further improvement in AMPs.

\section{METHODS}

Molecular modeling is a very much investigated technique for recognizing the potent compound without putting excessively exertion and investment in research [16-20]. ADT 1.5.6 software [21] is used by us to investigate the activity in terms of binding affinity ( $\mathrm{Kcal} / \mathrm{mol}$ ), and there after the outcomes are compared in binding affinity score for best-docked conformation. The structures of various AMPs were drawn by the help of ChemBiodraw ultra and further converted to the 3D structure using ChemBiodraw 3D. All the designed structures were optimized by energy minimization using MM2 method [22] and converted to readable format at the ADT interface. To identify the potential AMPs, a protein 3vma was selected which was downloaded from protein data bank [23]. The outcomes of results were analyzed by AutoDock Vina (ADT) result which reveals close contact, hydrogen bond, hydrophilic, and hydrophobic interactions. 


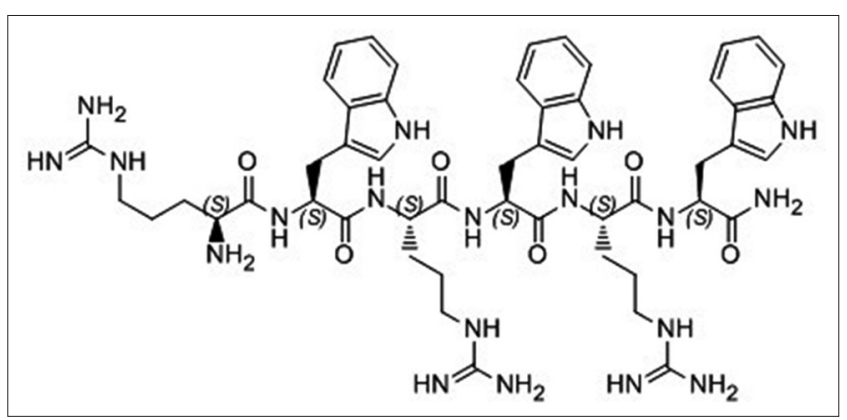

Fig. 1: MP196 an antimicrobial peptide

\section{RESULTS AND DISCUSSION}

We have designed the novel AMPs (KP_01R to KP_04S) on the basis of MP196 AMPs to find out the better lead compound. For the design of novel molecules, various structural changes were made on the MP196, i.e., the amino acids arginine (R) and tryptophan (W) at both terminals are replaced by leucine (L), phenylalanine (F) as well as reversing the stereochemistry of these amino acids to transform them as better ligands by keeping an amidation at C-terminal as depicted in (Fig. 2) [24].

Replacement of terminal amino acids leads to a change in the net charge as well as lipophilicity of the designed AMPs by using non-polar amino acids like leucine and phenylalanine. The designed molecules

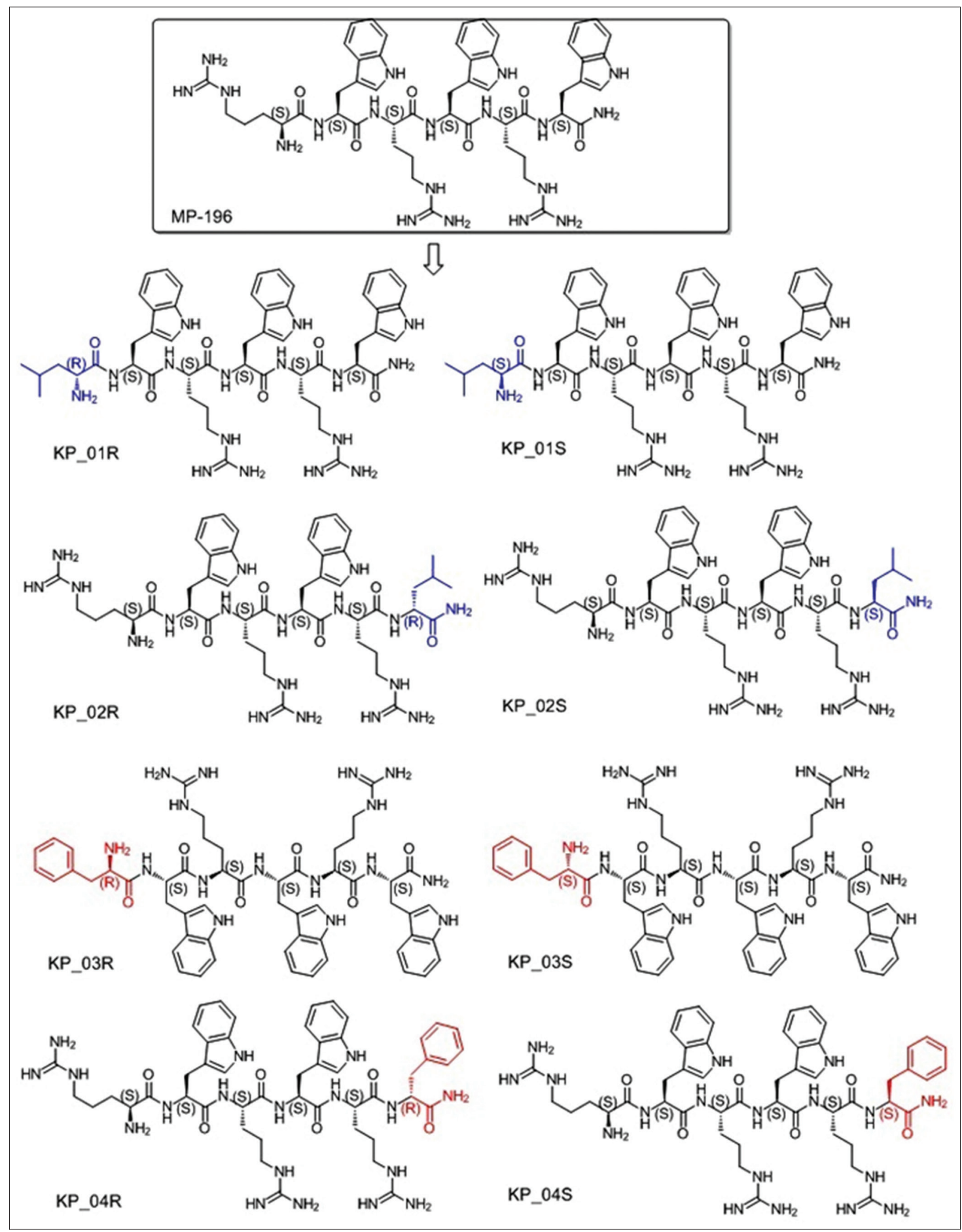

Fig. 2: Design of novel antimicrobial peptides-based MP196 
were analyzed to identify the most potent AMP by molecular modeling software, so selected 3vma protein of Escherichia coli was prepared by recognizing the binding site.

First, the validation of protein was done by extraction of ligand (Fig. 3) and after extraction of ligand from the protein; it was prepared for the docking study by adding the polar hydrogen, detecting root, and converting it to pdbqt extension file. For docking study, after extraction of ligand, 3vma protein is prepared by removing water molecules, repairing missing atoms, adding polar hydrogen only, and subsequently adding the Kollman charges. Further, the grid box was generated keeping the ligand as a center (Fig. 4).

From grid output file the configuration file "conf.txt" was prepared and command prompt was used for ADT molecular docking by giving command "program files \the scripps research institute\vina\vina. exe - config conf.txt - log log.txt" It generated the output file with the docking score or binding affinity (Kcal/mol), similarly, all the designed molecules were studied, and their binding affinities are showed in Table 1 .

The results revealed that all the designed AMPs showed better binding affinity ranging from -8.1 to $-9.0 \mathrm{Kcal} / \mathrm{mol}$ in comparison to MP196 AMP (-7.5 Kcal/mol, Table 1). Among them, KP_03R found to be best with $-9.0 \mathrm{Kcal} / \mathrm{mol}$ (Table 1 ), at 3vma, penicillin-binding protein of E. coli. In-depth analysis at the binding site of 3vma (Fig. 5), it is observed that KP_03R has two hydrogen bonds at the distance of 2.246 and 2.043 with the energy of -2.9 and $-6.1 \mathrm{Kcal} / \mathrm{mol}$, respectively (Fig. 6). The hydrogen bonds involved between the protein and KP_03R are hydrogen of Arg 286 and carbonyl of Asn 275 residue. Whereas, its antipode or other $S$ stereoisomer KP-03S showed a less binding affinity ( $-8.5 \mathrm{Kcal} / \mathrm{mol}$, Table 1$)$. Similarly, the stereochemistry affects the binding affinity of other designed AMPs suggesting that " $R$ " stereoisomer would be more effective than " $S$ " stereoisomers (Table 1). Further, we examined the various physical properties of the peptide by using the online peptide data bank using the basic amino acid sequence of KP_03R AMP. The amino acid residues sequence of this peptide was submitted to the AMP database (APD2) a predictive tool based on APD2 v2.34 (APD2; http://aps.unmc.edu/AP/main.php) [25,26]. The result

Table 1: Designed antimicrobial peptides based on MP196 and their binding energies

\begin{tabular}{ll}
\hline AMPs & Binding affinity (Kcal $\backslash \mathbf{m o l})$ \\
\hline MP196 & -7.5 \\
KP_01R & -8.8 \\
KP_01S & -8.5 \\
KP_02R & -8.9 \\
KP_02S & -8.3 \\
KP_03R & -9.0 \\
KP_03S & -8.5 \\
KP_04R & -8.2 \\
KP_04S & -8.1 \\
\hline
\end{tabular}

AMPs: Antimicrobial peptides

Table 2: Calculated peptide parameters and the APD-based prediction of properties of KP_03R (FWRWRW-NH2)

\begin{tabular}{ll}
\hline Parameters & Values \\
\hline Amino acids residues & F-W-R-W-R-W-NH \\
Percentage of each amino acid & Phe (F) ratio $=16 \%$ \\
& Trp (W) ratio $=50 \%$ \\
Hydrophobic ratio & Arg (R) ratio $=33 \%$ \\
Total net charge & $66 \%$ \\
GRAVY & +2 \\
\hline
\end{tabular}

GRAVY: Grand average hydropathy value of the peptide, APD: Antimicrobial peptide database

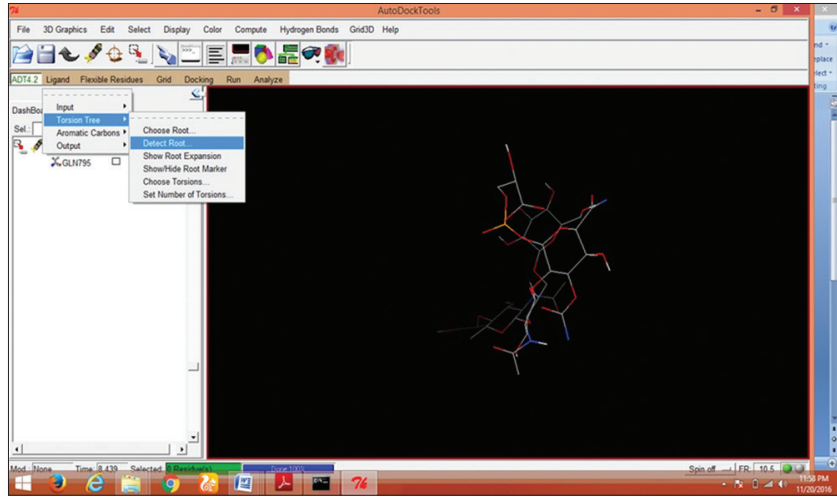

Fig. 3: Extracted ligand from 3vma protein

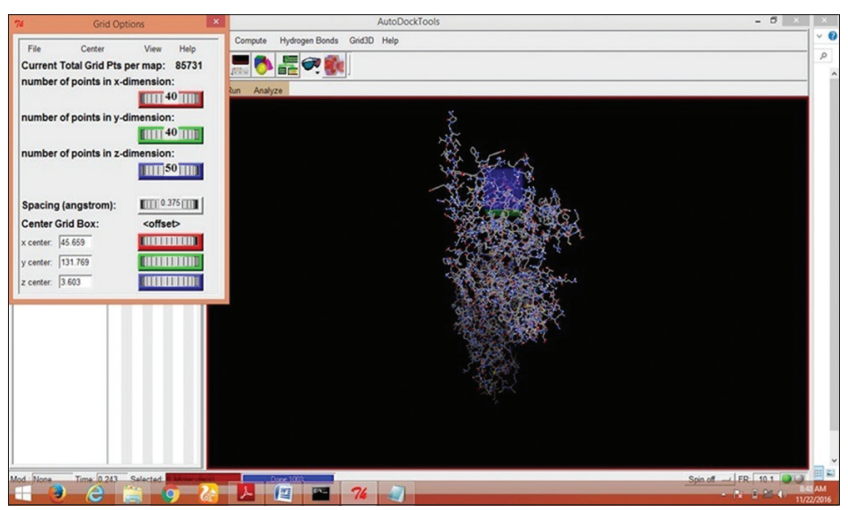

Fig. 4: Set grid box on 3vma protein as a macromolecule

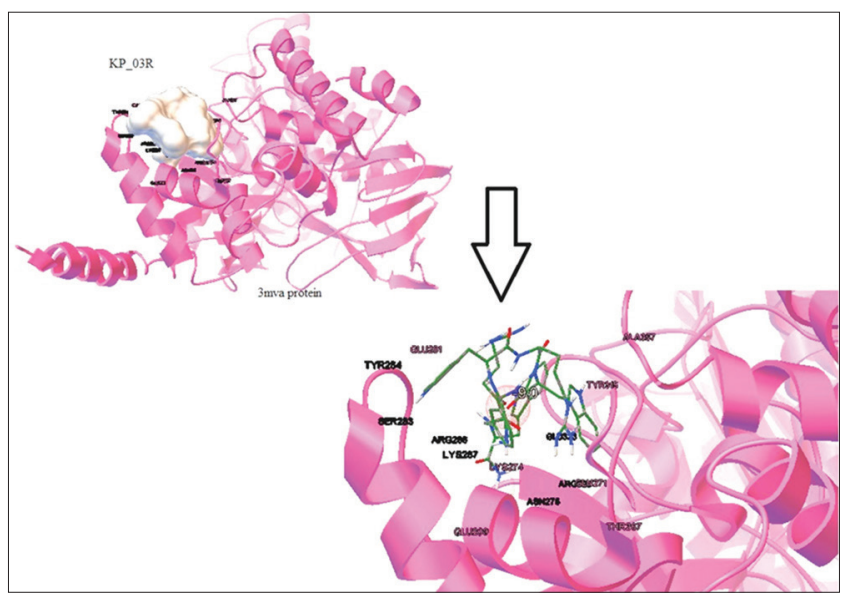

Fig. 5: Close contacts of KP_03R (atom color in ball and stick model) with neighboring amino acid residues of $3 \mathrm{vma}$ protein (pink color in ribbon structure)

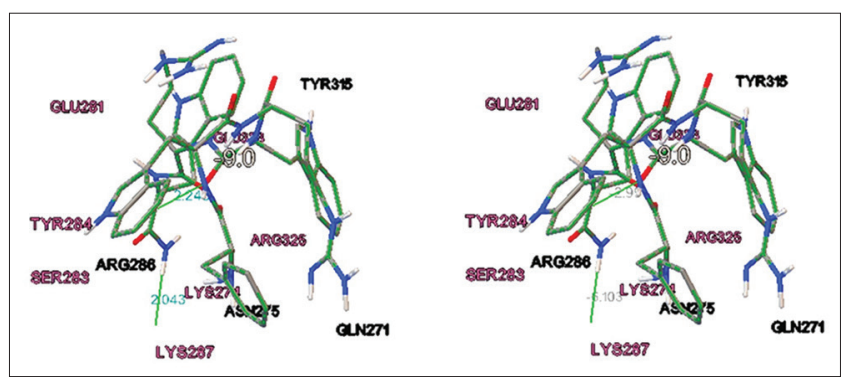

Fig. 6: Visualization of active binding sites of 3 vam protein and interaction with KP_03R (a) showed the distance of hydrogen bond and (b) showed hydrogen bond energies 
in Table 2 showed optimum hydrophobicity with $66 \%$, total net charge +2 and grand average hydropathy value of the peptide -1.48 for KP_03R suggesting that it could be a potent AMP.

\section{CONCLUSION}

Antimicrobial drug resistance is a major concern in current and AMP could serve a better alternate to overcome it. MP196 a short chain consisting six amino acids AMP was selected for further improvement with modifying the terminal amino acid as well as their stereochemistry. The eight different AMPs were designed, and among them, KP_03R (FWRWRW- $\mathrm{NH}_{2}$ ) showed the maximum activity against 3vma protein (A penicillin-binding protein from $E$. coli.) with the binding affinity of $-9.0 \mathrm{Kcal} \backslash \mathrm{mol}$. Moreover, the comparison was also made between $R$ and $S$ stereoisomers of the peptides. The $S$-stereoisomer KP-03S proved to be less active than $R$-stereoisomer KP_03R against 3vma receptor protein. Further investigations on these novel AMPs will provide the promising tool for new drug development to treat microbial infection and provide an alternative in case of drug resistance.

\section{ACKNOWLEDGMENTS}

The author is grateful to SERB-Department of Science and Technology Government of India for research funding.

\section{REFERENCES}

1. Gordon YJ, Romanowski EG, McDermott AM. A review of antimicrobial peptides and their therapeutic potential as anti-infective drugs. Curr Eye Res 2005;30(7):505-15.

2. Jenssen H, Hamill P, Hancock RE. Peptide antimicrobial agents. Clin Microbiol Rev 2006;19(3):491-511.

3. Hancock RE, Scott MG. The role of antimicrobial peptides in animal defenses. Proc Natl Acad Sci U S A 2000;97(16):8856-61.

4. Dubos RJ. Studies on a bactericidal agent extracted from a soil Bacillus: II. Protective effect of the bactericidal agent against experimental Pneumococcus infections in mice. J Exp Med 1939;70(1):11-7.

5. Hotchkiss RD, Dubos RJ. Fractionation of the bactericidal agent from cultures of a soil Bacillus. J Biol Chem 1940;132:791-2.

6. Van Epps HL. René Dubos: Unearthing antibiotics. J Exp Med 2006;203(2):259.

7. Dubos RJ, Hotchkiss RD. The production of bactericidal substances by aerobic Sporulating bacilli. J Exp Med 1941;73(5):629-40.

8. Rammelkamp CH, Weinstein L. Toxic effects of tyrothricin, gramicidin and tyrocidine. J Infect Dis 1942;71:166-73.

9. Ohtani S, Okada T, Yoshizumi H, Kagamiyama H. Complete primary structures of two subunits of purothionin A, a lethal protein for brewer's yeast from wheat flour. J Biochem 1977;82(3):753-67.

10. Hirsch JG. Phagocytin: A bactericidal substance from polymorph nuclear leucocytes. J Exp Med 1956;103(5):589-611.

11. Groves ML, Peterson RF, Kiddy CA. Poliomorphism in the red protein isolated from milk of individual cows. Nature 1965;207(5000):1007-8.

12. Zeya HI, Spitznagel JK. Antibacterial and enzymic basic proteins from leukocyte lysosomes: Separation and identification. Science 1963;142(3595):1085-7.

13. Chantson JT, Verga Falzacappa MV, Crovella S, MetzlerNolte N. Solid-phase synthesis, characterization, and antibacterial activities of metallocene-peptide bioconjugates. ChemMedChem 2006;1(11):1268-74

14. Strøm MB, Haug BE, Skar ML, Stensen W, Stiberg T, Svendsen JS. The pharmacophore of short cationic antibacterial peptides. J Med Chem 2003;46(9):1567-70.

15. Albada HB, Chiriac AI, Wenzel M, Penkova M, Bandow JE, Sahl HG, et al. Modulating the activity of short arginine-tryptophan containing antibacterial peptides with $\mathrm{N}$-terminal metallocenoyl groups. Beilstein J Org Chem 2012;8:1753-64

16. Chaurasiya S, Kaur P, Nayak SK, Khatik GL. Virtual screening for identification of novel potent EGFR inhibitors through AutoDock Vina molecular modeling software. J Chem Pharm Res 2016;8:353-60.

17. Shruthy VS, Shakkeela Y. In silico design, docking, synthesis, and evaluation of thiazole Schiff bases. Int J Pharm Pharm Sci 2014;6:271-5.

18. Muthukala B, Kanakarajan S, Kamalanathan A. In silico docking of quercetin compound against the HeLa cell line proteins. Int J Curr Pharm Res 2015;7:13-6.

19. Munusami P, Vasavi C, Divya G. Molecular docking studies on flavonoid compounds: An insight into aromatase inhibitors. Int J Pharm Pharm Sci 2014;6:141-8.

20. Kaur P, Khatik GL. Advancements in non-steroidal antiandrogens as potential therapeutic agents for the treatment of prostate cancer. Mini Rev Med Chem 2016;16(7):531-46.

21. Trott O, Olson AJ. AutoDock Vina: Improving the speed and accuracy of docking with a new scoring function, efficient optimization, and multithreading. J Comput Chem 2010;31:455-61.

22. Energy Minimizations were Performed MM2 Interface Program on ChemBio3D Ultra 12.0, and Structures were Drawn by ChemBioDrwa Ultra 12.0. Cambridge: Cambridge Soft; 1985.

23. Available from: http://www.rcsb.org/pdb/explore.do?pdbId=3vma. [Last accessed on 2016 Sep 22].

24. Wenzel M, Chiriac AI, Otto A, Zweytick D, May C, Schumacher C, et al. Small cationic antimicrobial peptides delocalize peripheral membrane proteins. Proc Natl Acad Sci U S A 2014;111(14):E1409-18.

25. Available from: http://www.aps.unmc.edu/AP/prediction/prediction main.php. [Last accessed on 2016 Nov 25].

26. Wang G, Li X, Wang Z. APD2: The updated antimicrobial peptide database and its application in peptide design. Nucleic Acids Res 2009;37:D933-7 